\title{
PROBLEMÁTICA DE LA APLICACIÓN DE AGUA MARINA DESALINIZADA AL RIEGO AGRÍCOLA. CASO DE ESTUDIO DEL CANAL DEL CAMPO DE CARTAGENA
}

\author{
Martínez-Alvarez, V. ${ }^{1}$, Martin-Gorriz, $B{ }^{1}{ }^{1}$, Soto-Garcia, M. ${ }^{2}$
}

1 Escuela Técnica Superior de Ingeniería Agronómica, Universidad Politécnica de Cartagena, Paseo Alfonso XIII, 48. 30203 Cartagena. E-mail: victoriano.martinez@upct.es y b.martin@upct.es.

2 Comunidad de Regantes del Campo de Cartagena, Paseo Alfonso XIII, 22, 30201 Cartagena. E-mail: mariano.soto@crcc.es

\section{Resumen}

En este trabajo se presenta una revisión de las principales cuestiones agronómicas y de manejo que se deben considerar cuando se plantea la incorporación de agua marina desalinizada en el riego agrícola. Este análisis se fundamenta en una revisión bibliográfica a escala internacional de las primeras experiencias que se están dando a conocer mediante publicaciones en el ámbito científico.

Los principales aspectos a considerar en el riego con agua marina desalinizada son: (1) carencias y desequilibrios nutricionales en la composición química, que pueden limitar el desarrollo de los cultivos; (2) elevada concentración de boro, que puede producir problemas de fitotoxicidad en cultivos sensibles; (3) elevada acidez y poder corrosivo como consecuencia de la escasa mineralización del agua, que puede afectar a las instalaciones de riego; y (4) necesidad de una mayor capacidad de control y gestión del agua en las explotaciones agrícolas.

Además, se analiza la problemática en un caso de estudio concreto, que se corresponde con la incorporación de agua marina desalinizada al embalse de La Pedrera, analizándose la mitigación de sus posibles inconvenientes mediante la mezcla en distintas proporciones con el agua continental suministrada habitualmente a dicho embalse mediante las infraestructuras del postrasvase Tajo-Segura.

\section{1- Introducción y objetivos}

La incorporación de agua marina desalinizada (AMD) a zonas regables del sureste español es la principal estrategia recogida en la planificación hídrica española con el fin de hacer frente al déficit estructural de agua, que persiste en esta zona desde hace varias décadas.

La revisión a escala global de las principales experiencias de riego agrícola con agua desalinizada pone de manifiesto que en numerosos países con clima árido o semiárido, y que además disponen de una agricultura altamente tecnificada, la desalinización de aguas salobres representa una fuente de agua suplementaria desde hace varias décadas. Sin embargo, a pesar de que hay países donde se está considerando la posibilidad de aplicar AMD al riego agrícola en un futuro próximo, tras la revisión de las bases de datos internacionales más relevantes sólo se han encontrado referencias de su utilización en España e Israel. En España las referencias existentes son: (1) las Islas Canarias, donde hay una prolongada experiencia cuyas conclusiones no son extrapolables al sureste español 
dada su gran singularidad agroambiental y en el manejo de los cultivos, y (2) recientes suministros desde plantas desalinizadoras del sureste, que generalmente se han mezclado de forma minoritaria con otros recursos continentales y han carecido de seguimiento científico. La experiencia israelí, que se ha desarrollado durante la última década, se caracteriza por un adecuado seguimiento científico y por su semejanza agronómica con el sureste español, motivo por el que sus resultados y conclusiones representan una fuerte de información fundamental para abordar con éxito la incorporación de AMD al riego agrícola.

La principal ventaja del AMD es su condición de recurso hídrico inagotable y no sujeto a variaciones climáticas, por lo que estratégicamente resulta idóneo para aumentar de forma sistemática la disponibilidad de recursos hídricos para riego agrícola en zonas deficitarias como el sureste español. Como principal inconveniente persiste el elevado consumo energético asociado a su producción, que cuadriplica el del trasvase Tajo-Segura, generando un coste del agua producto muy elevado y un nivel de emisiones de gases de efecto invernadero poco compatible con las políticas demandadas para el control del cambio climático. El coste total de producción en las desalinizadoras de agua marina de la cuenca del Segura oscila entre 0,60 y $0,69 € \mathrm{~m}^{-3}$, mientras que el valor marginal neto del agua de riego en las zonas regables de la costa se sitúa entre 0,51 y $1,05 € \mathrm{~m}^{-3}$. Estos datos ponen de manifiesto que, con los costes actuales, no se puede generalizar la idea de suplir la actual carencia de recursos hídricos para riego del sureste español con AMD. Solamente los cultivos más tecnificados y con mayores márgenes económicos pueden soportar los costes del AMD, pero a costa de una notable pérdida de rentabilidad en comparación con la situación actual. En este sentido también se manifestaron los expertos convocados por la FAO en 2004, donde se concluyó que la aplicación de técnicas de desalinización al riego agrícola es, en general, poco efectiva económicamente, limitándose su utilización a casos concretos de cultivos con alto valor añadido y subvenciones gubernamentales (FAO, 2006).

La osmosis inversa se ha generalizado como la tecnología de referencia para la desalinización de agua marina, ya que presenta consumos energéticos y costes de producción reducidos en comparación con el resto de tecnologías aplicables a gran escala. El agua resultante de esta técnica (agua marina osmotizada) se caracteriza por su escasa mineralización e importantes desequilibrios en su composición, por lo que no es apta para ningún tipo de suministro (doméstico, agrario o industrial). Para adecuar las características del agua marina osmotizada a los requerimientos de los distintos usos debe someterse a postratamientos de remineralización en la propia planta desalinizadora, o mezclarse con otras aguas que corrijan sus desequilibrios. Los postratamientos aplicados actualmente se han diseñado con el fin de adecuar las características del agua resultante (agua marina desalinizada, AMD) a los requerimientos de calidad para consumo humano, recogidos en el RD 140/2003. Sin embargo, estos requerimientos no son los más adecuados para la agricultura, por lo que el uso de AMD conforme a los criterios de calidad del RD 140/2003 en el riego agrícola puede derivar en problemas agronómicos que afecten tanto a la productividad de los cultivos como a la calidad de las cosechas.

Estos problemas agronómicos ya se han puesto de manifiesto en Israel (Yermiyahu et al., 2007; Ben-Gal et al., 2009), donde las primeras experiencias de sustitución de agua de origen continental por AMD proveniente de las plantas de Ashkelon y Palmachim no están siendo plenamente satisfactorias. En estas experiencias se han detectado problemas agronómicos que afectan a la productividad de los cultivos, a los costes de fertirrigación y a la conservación de los suelos agrícolas, aspectos que pueden comprometer su viabilidad económica en el corto plazo y su sostenibilidad ambiental en el medio-largo plazo.

En este trabajo se presenta una revisión de las principales cuestiones agronómicas y de manejo que se deben considerar cuando se plantea la incorporación de AMD en el riego agrícola. Este análisis se fundamenta en una revisión bibliográfica a escala internacional de 
las primeras experiencias que se están dando a conocer mediante publicaciones en el ámbito científico. Además, se analiza la problemática en un caso de estudio concreto, que se corresponde con la incorporación de AMD al embalse de La Pedrera, analizándose la mitigación de sus posibles inconvenientes mediante la mezcla en distintas proporciones con el agua continental suministrada habitualmente a dicho embalse mediante las infraestructuras del postrasvase Tajo-Segura.

\section{2- Aspectos agronómicos a considerar en la aplicación de agua marina desalinizada al riego agrícola}

La incorporación de AMD a la agricultura puede producir efectos agronómicos muy diferentes, dependiendo de la calidad del agua de riego que se sustituye. Cuando el AMD, cuya conductividad eléctrica (CE) es generalmente inferior a $0,5 \mathrm{dS} \mathrm{m}^{-1}$, sustituye a aguas de riego de baja calidad, se puede esperar un aumento de la productividad y la calidad del rendimiento de los cultivos debido a la reducción de estrés por salinidad. Por otra parte, se puede conseguir una disminución significativa en las necesidades de riego, ya que la fracción de lavado se puede reducir drásticamente. Algunos ejemplos de estos efectos beneficiosos se han documentado para diferentes cultivos en España (Zarzo et al., 2013) e Israel (Ben-Gal et al., 2009).

Por otro lado, cuando se sustituyen aguas de buena calidad, la reducción de la CE del AMD no implica beneficio agronómico alguno, mientras que conlleva algunos riesgos agronómicos. Además de su baja mineralización, el AMD se caracteriza por una composición química muy diferente a las de las fuentes de agua convencionales. En la composición de sales del AMD predominan los iones sodio $\left(\mathrm{Na}^{+}\right)$y cloruro $\left(\mathrm{Cl}^{-}\right)$, presentando muy baja concentración de otros minerales como el calcio $\left(\mathrm{Ca}^{2+}\right)$, el magnesio $\left(\mathrm{Mg}^{2+}\right)$ y el sulfato $\left(\mathrm{SO}_{4}{ }^{2-}\right)$, así como alta concentración de elementos fitotóxicos como el boro (B). Por lo tanto, se puede afirmar que existe un problema agronómico asociado a los suministros de AMD para regadío, que debe abordarse específicamente, y que puede condicionar de forma sustancial la gestión del agua en la agricultura.

\section{1- Carencia de nutrientes esenciales y efectos en la fertirrigación}

Las aguas naturales, junto con el contenido mineral del suelo, generalmente proporcionan niveles de nutrientes esenciales como $\mathrm{Ca}^{2+}, \mathrm{Mg}^{2+}$ y $\mathrm{SO}_{4}{ }^{2-}$ suficientes para evitar su inclusión en los programas de fertilización. Por el contrario, las concentraciones de estos minerales en el AMD son significativamente inferiores a los valores recomendados para el riego agrícola, ya que el proceso de osmosis inversa no sólo separa las sales indeseables del agua, sino que también elimina minerales que son nutrientes esenciales para el crecimiento vegetal. Por lo tanto, la sustitución de las fuentes de agua convencionales por AMD puede limitar el correcto desarrollo de los cultivos, afectando a la calidad y rendimiento de la producción, como ya ha ocurrido con cultivos de tomate, albahaca y flores en Israel (Yermeyahu et al., 2007; Ben-Gal et al., 2009). Para garantizar las necesidades nutricionales de los cultivos, la falta de nutrientes esenciales $\left(\mathrm{Ca}^{2+}, \mathrm{Mg}^{2+}\right.$ y $\mathrm{SO}_{4}{ }^{2-}$ ) puede remediarse mediante la adecuación de los programas de fertilización, pero representa un aumento en los costes de producción para los agricultores.

La Tabla 1 muestra los requerimientos nutricionales de $\mathrm{Ca}^{2+}, \mathrm{Mg}^{2+}$ y $\mathrm{SO}_{4}^{2-}$ característicos de los cultivos hortícolas del sureste español, así como la concentración típica de estos nutrientes en las aguas de riego convencionales del sureste español e Israel, y sus concentraciones en el AMD de varias plantas desalinizadoras de agua marina para suministro agrícola. Se observa que las necesidades de los cultivos están siendo satisfechas actualmente por el agua de riego, sobre todo en el sureste español. Es evidente que si se 
sustituyen estas aguas convencionales de riego por AMD habrá que incorporar una parte importante de las necesidades de $\mathrm{Ca}^{2+}, \mathrm{Mg}^{2+}$ y $\mathrm{SO}_{4}{ }^{2-}$ mediante fertilizantes, especialmente en suelos de escasa mineralización y en cultivos sin suelo. En este sentido, Ben-Gal et al. (2009) indican que este incremento en el coste de fertirrigación es de $3.500 \$$ ha $^{-1}$ para cultivo de pimiento en invernadero.

Por lo tanto, cuando se planifica aplicar AMD en la agricultura es necesario reincorporar estos nutrientes esenciales en el agua de riego, planteándose tres posibles alternativas: (1) se pueden añadir en los postratamientos de la planta desalinizadora; (2) pueden ser añadidos por los agricultores mediante fertilizantes; o (3) se pueden incorporar mediante la mezcla del AMD con otras aguas naturales de elevada mineralización. Estas posibles opciones deben evaluarse económicamente ya que dependiendo de la estrategia seleccionada los costes de remineralización se trasladan de los productores de AMD a los agricultores.

Tabla 1. Requerimientos nutricionales de $\mathrm{Ca}^{2+}, \mathrm{Mg}^{2+}$ y $\mathrm{SO}_{4}{ }^{2-}$ característicos de los cultivos hortícolas, concentración típica de estos nutrientes en las aguas de riego convencionales del sureste español e Israel, y en el AMD de varias plantas desalinizadoras.

\begin{tabular}{|c|c|c|c|}
\hline Cultivos & $\begin{array}{l}\text { Necesidades de } \\
\mathrm{Ca}^{2+}\left(\mathrm{mg} \mathrm{L}^{-1}\right)\end{array}$ & $\begin{array}{l}\text { Necesidades de } \\
\mathrm{Mg}^{2+}\left(\mathrm{mg} \mathrm{L}^{-1}\right)\end{array}$ & $\begin{array}{l}\text { Necesidades de } \\
\mathrm{SO}_{4}{ }^{2-}\left(\mathrm{mg} \mathrm{L}^{-1}\right)\end{array}$ \\
\hline Cultivos hortícolas (1) & $80-120$ & $24-36$ & $100-150$ \\
\hline $\begin{array}{l}\text { Aguas de riego } \\
\text { convencionales }\end{array}$ & $\begin{array}{c}\text { Concentración de } \\
\mathrm{Ca}^{2+}\left(\mathrm{mg} \mathrm{L}^{-1}\right)\end{array}$ & $\begin{array}{l}\text { Concentración } \\
\text { de } \mathrm{Mg}^{2+}\left(\mathrm{mg} \mathrm{L}^{-1}\right)\end{array}$ & $\begin{array}{c}\text { Concentración de } \\
\mathrm{SO}_{4}{ }^{2-}\left(\mathrm{mg} \mathrm{L}^{-1}\right)\end{array}$ \\
\hline Sureste de España(2) & $90-110$ & $35-45$ & $200-350$ \\
\hline Israel (3) & $45-60$ & $20-25$ & $60-80$ \\
\hline $\begin{array}{l}\text { AMD de diferentes } \\
\text { plantas }\end{array}$ & $\begin{array}{c}\text { Concentración de } \\
\mathrm{Ca}^{2+}\left(\mathrm{mg} \mathrm{L}^{-1}\right)\end{array}$ & $\begin{array}{c}\text { Concentración } \\
\text { de } \mathrm{Mg}^{2+}\left(\mathrm{mg} \mathrm{L}^{-1}\right)\end{array}$ & $\begin{array}{l}\text { Concentración de } \\
\mathrm{SO}_{4}^{2-}\left(\mathrm{mg} \mathrm{L}^{-1}\right)\end{array}$ \\
\hline AMD1 (4) & 29 & 4,3 & 6,6 \\
\hline AMD2 (5) & 4,2 & 5,5 & 14,4 \\
\hline AMD3 (6) & $32-34$ & 0 & 0 \\
\hline AMD4 (7) & $40-46$ & 0 & $60-80$ \\
\hline
\end{tabular}

\footnotetext{
(1) Datos representativos para cultivos hortícolas en el sureste español

(2) Datos de cerca de 50 comunidades de regantes del sureste español

(3) Yermiyahu et al. (2007)

(5) Planta desalinizadora de Torrevieja, datos de Julio de 2014.

(6) Planta desalinizadora de Punta de los Vientos (Islas Canarias, Díaz et al., 2013)

(7) Planta desalinizadora de Hedara en Israel (Lahav et al., 2010)

(8) Planta desalinizadora de Ashkelon en Israel (Yermiyahu et al., 2007)
}

\section{2- Fitotoxicidad por boro}

El boro es un micronutriente esencial para las plantas, que necesitan pequeñas cantidades para su crecimiento y desarrollo, pero que puede llegar a ser muy tóxico si la cantidad es ligeramente superior a la requerida. Los efectos fisiológicos adversos del exceso de boro en las plantas se han revisado extensamente (Hilal et al., 2011), implicando la reducción de la división celular, con el consiguiente retraso en el crecimiento de brotes y 
raíces, la inhibición de la fotosíntesis, la deposición de lignina y suberina, la disminución de la clorofila en las hojas, etc.

La concentración de boro en el AMD es mayor que en aguas convencionales, de tal manera que el riego con AMD puede aumentar el contenido de boro del suelo sustancialmente, provocando problemas de toxicidad que conducen a la disminución de rendimientos en cultivos sensibles, como ha ocurrido en Israel con concentraciones de boro de 0,6, 1,2 y 2,0 $\mathrm{mg} \mathrm{L}^{-1}$ (Yermeyahu et al., 2007). El alto contenido de boro en el AMD se debe a (1) su alta concentración en el agua marina $\left(4,5-6 \mathrm{mg} \mathrm{L}^{-1}\right)$ en relación con las aguas naturales ( 0 a $\left.1,5 \mathrm{mg} \mathrm{L}^{-1}\right)$; y (2) el hecho de que una gran parte de boro en el agua de mar toma la forma de ácido bórico $\left(\mathrm{H}_{3} \mathrm{BO}_{3}\right)$, sin carga iónica, por lo que presenta menor separación en las membrana de osmosis inversa que los iones. Por lo tanto, es necesario considerar tecnologías específicas para la reducción de boro en plantas desalinizadoras para la agricultura, como la incorporación de una segunda etapa de osmosis inversa o el uso de resinas de intercambio iónico. En ambos casos, la inversión y el coste por metro cubico de AMD aumenta (Shaffer et al., 2012).

Hay distintas clasificaciones de los cultivos en función de su tolerancia al boro (Hilal et al., 2011). La regulación del contenido de boro en el AMD varía en cada país. La recomendación israelí $\left(0,3 \mathrm{mg} \mathrm{L}^{-1}\right)$ protegería incluso los cultivos más sensibles, mientras que en España el requisito de las nuevas plantas desalinizadoras del Programa AGUA es de $0,5 \mathrm{mg} \mathrm{L}^{-1}$ (Zarzo et al., 2013), que es claramente un requisito del sector agrícola debido a que la norma de agua potable en España es de $1 \mathrm{mg} \mathrm{L}^{-1}$. Este límite de $0,5 \mathrm{mg} \mathrm{L}^{-1}$ puede resultar inadecuado para algunos cultivos del sureste español como los cítricos.

\section{3- Fitotoxicidad por $\mathrm{Cl}^{-}$y $\mathrm{Na}^{+}$}

En el agua marina el $55 \%$ y $31 \%$ de contenido de sal son $\mathrm{Cl}^{-}$y $\mathrm{Na}^{+}$, respectivamente. Después del proceso de osmosis inversa estos iones todavía predominan en la composición del AMD y pueden producir fitotoxicidad en cultivos sensibles. La mayoría de los cultivos leñosos son sensibles a concentraciones elevadas $\mathrm{Cl}^{-}$y $\mathrm{Na}^{+}$, mientras que los cultivos anuales no son tan sensibles.

El $\mathrm{Cl}^{-}$y $\mathrm{Na}^{+}$también pueden ser absorbidos directamente por las hojas durante el riego por aspersión, causando daño foliar. El riesgo de toxicidad al $\mathrm{Na}^{+}$se reduce si el agua de riego presenta altas concentraciones de $\mathrm{Ca}^{2+}$, por lo que una evaluación razonable de la toxicidad potencial al $\mathrm{Na}^{+}$debe realizarse utilizando la relación de adsorción de sodio (RAS) del agua de riego, según se analiza en el siguiente epígrafe.

Las concentraciones de $\mathrm{Cl}^{-}$y Na+ en el AMD de plantas españolas $\left(0,56 \mathrm{mg} \mathrm{L}^{-1}\right.$ para AMD1 y $0,76 \mathrm{mg} \mathrm{L}^{-1}$ para AMD2, Tabla 1) representan un riesgo moderado que podría afectar a los cultivos sensibles en su área de influencia. Los valores en las plantas israelíes son mucho más bajos (por debajo de $0,3 \mathrm{mg} \mathrm{L}^{-1}$ en DSW3 y DSW4, la Tabla 1), de acuerdo con los valores máximos recogidos en las recomendaciones israelíes.

Experiencias a medio-largo plazo con AMD en Lanzarote (Díaz et al., 2013) indican un aumento significativo en los niveles de salinidad y la concentración de $\mathrm{Na}^{+}$en el suelo tras un período de riego de 20 años, lo que indica que la sostenibilidad a largo plazo de estos sistemas de cultivo requiere (1) sustancial mejoras en la calidad AMD y (2) el aumento de las fracciones de lavado en la programación del riego para evitar acumulaciones de sal en la zona radicular de los cultivos. 


\section{4- Riesgo de sodificación de suelos}

Entre los riesgos potenciales asociados al riego con AMD está la degradación de la estructura del suelo por alcalinización, que afecta en gran medida al rendimiento de los cultivos. Aparte de los efectos directos por fitotoxicidad, la concentración de $\mathrm{Na}^{+}$puede producir efectos adversos en las propiedades físicas del suelo en forma de dispersión de arcilla, dando lugar al deterioro de la estabilidad de los agregados; a la disminución de la conductividad hidráulica del suelo; al aumento de la formación de costras en superficie, incrementando la escorrentía y la erosión del suelo; a la compactación del suelo; y a la disminución de la aireación del suelo (Muyen et al., 2011).

Un indicador cuantitativo del riesgo de sodificación del suelo es la relación de adsorción de sodio (RAS), que se calcula a partir de las concentraciones de $\mathrm{Na}^{+}, \mathrm{Ca}^{2+}$ y $\mathrm{Mg}^{2+}$ en el agua de riego. Los valores de RAS y la CE deben considerarse simultáneamente para evaluar el riesgo de sodificación del suelo (Ayers y Westcot, 1985).

EI RAS en agua marina está por encima de 10. El agua marina osmotizada presenta altas concentraciones de $\mathrm{Na}^{+}$, junto con muy baja o ninguna de $\mathrm{Ca}^{2+} \mathrm{y} \mathrm{Mg}^{2+}$, por lo que generalmente resulta en valores del RAS entre 9 y 10. Los post-tratamientos en planta desalinizadora pueden añadir $\mathrm{Ca}^{2+} \mathrm{y} \mathrm{Mg}^{2+}$, disminuyendo el RAS en función de su intensidad. Para aguas de riego con baja concentración de sólidos disueltos totales, como ocurre con el AMD, se recomienda un RAS < 3 (Ayers y Westcot, 1985). En las plantas desalinizadoras de agua marina españolas el RAS se encuentra alrededor de 6 (5.5 para AMD1 y 6.8 para AMD2, Tabla1), implicando un riesgo de sodificación del suelo moderado. En cuanto a las plantas de desalinización de Israel, el restrictivo umbral de concentración de $\mathrm{Na}^{+}$recogido en sus recomendaciones para uso agrícola de AMD conduce a valores de RAS cercanos a cero (0.4 para AMD3 y 0.5 para AMD4, Tabla 1$)$, lo que minimiza el riesgo de sodificación de suelos. A partir del conocimiento acumulado en Israel (Lahav et al., 2010), parece que el riego con valores de RAS de entre 4 y 5,5 es probablemente aceptable (aunque no ideal), mientras que el uso a largo plazo de los valores de RAS de 60 superiores dará lugar a un deterioro de las propiedades del suelo.

Por lo tanto, el riego con AMD requiere una vigilancia continua del suelo para detectar cualquier degradación de su estructura. Si se presenta el problema, debe ser abordado con prácticas de manejo adecuadas, tales como el aumento de la fracción de lavado en la programación del riego o la aplicación de enmiendas de $\mathrm{Ca}^{2+}$. Cabe señalar que el riesgo de sodificación es más importante en suelos con contenido significativo de arcilla, mientras que en suelos arenosos el valor del RAS es menos crítico. Del mismo modo, en las zonas donde el suelo se lava regularmente por el agua de lluvia, el impacto de valores altos del RAS es menor (Duranceau et al., 2011).

\section{5- Baja alcalinidad y capacidad tampón}

La alcalinidad es una medida de la capacidad tampón del agua, es decir, de la capacidad de las bases para neutralizar los ácidos. Las aguas con baja alcalinidad son muy susceptibles a cambios en el $\mathrm{pH}$ ya que carecen de capacidad tampón. La alcalinidad también está directamente relacionada con la dureza del agua, que se determina por la concentración de cationes multivalentes $\left(\mathrm{Ca}^{2+}\right.$ y $\left.\mathrm{Mg}^{2+}\right)$.

Las membranas de ósmosis inversa son muy eficientes en la eliminación de bicarbonatos y carbonatos, así el agua marina osmotizada se caracteriza por un $\mathrm{pH}$ muy bajo y prácticamente nula alcalinidad y dureza del agua, llegando a ser incluso agresiva hacia los componentes de los sistemas de distribución de agua (Duranceau et al., 2011). Para evitar la inestabilidad química del AMD antes de su incorporación a los sistemas de 
suministro, se debe incrementar la alcalinidad y dureza del agua en la etapa de posttratamiento (Birnhack et al., 2010).

Se recomiendan valores de alcalinidad altos en el AMD para riego agrícola por varias razones: (1) para reducir la corrosión en los elementos metálicos de los sistemas de distribución, tales como tubos de metal, válvulas y dispositivos; (2) para evitar la corrosión de las tuberías, que produce mayores pérdidas de carga y descarga de los iones metálicos en el agua; y (3) para estabilizar el pH cuando se añadan fertilizantes ácidos o básicos. Esta última cuestión es especialmente relevante para la agricultura, ya que el AMD puede causar cambios repentinos en el pH durante la adición de fertilizantes y puede tener un profundo impacto en la disponibilidad de nutrientes y, en última instancia, en la productividad agrícola (Yermeyahu et al., 2007). Por otra parte, en los sistemas de riego que han distribuido aguas duras durante largos períodos la formación de incrustaciones de carbonato cálcico es habitual, y el cambio a aguas excesivamente blandas podría desprender estas incrustaciones, afectando a filtros y contadores volumétricos, e incluso provocando el bloqueo de la tubería.

Por todas las razones anteriores, el criterios de calidad para uso agrícola del AMD de Israel establece un nivel de alcalinidad mínima recomendada de $80 \mathrm{mg} \mathrm{L}^{-1} \operatorname{como~}^{\mathrm{CaCO}} 3$, y un pH lo más alto posible, aunque siempre por debajo de 8,5 (Lahav y Birnhack, 2007). Además, para controlar el potencial del AMD para precipitar o disolver carbonato cálcico, se recomienda un Potencial de Precipitación de Carbonato de Calcio (CCPP) entre 3 y 10, lo que significa un ligero potencial de precipitación de carbonatos, garantizando la estabilidad de las incrustaciones existentes.

Las aguas AMD consideradas en este estudio (AMD1, AMD2, AMD3, AMD4) presentan valores de $\mathrm{pH}$ adecuados para la agricultura, pero con unos niveles de alcalinidad que generalmente están por debajo de las recomendaciones israelíes, lo que indica que los procesos de post-tratamiento deben intensificarse en los suministros de AMD para uso agrícola.

\section{3- Caso de estudio del Canal del Campo de Cartagena}

Actualmente se está produciendo la adquisición de AMD por parte de comunidades de regantes del interior de la cuenca del Segura, y la consiguiente solicitud de su intercambio por derechos del trasvase Tajo-Segura correspondientes a los usuarios del Canal del Campo de Cartagena en el embalse de La Pedrera. Se ha realizado una modelización predictiva de la calidad del agua de riego en el embalse de La Pedrera bajo distintos escenarios de mezcla, tomando como datos de partida las analíticas de la planta desalinizadora de Torrevieja y del agua con que se abastece La Pedrera. Se han modelizado las mezclas considerando proporciones de cada una de las aguas entre el 0 y $100 \%$, con incrementos del 10\% (Fig. 1). La modelización se ha realizado con el programa PHREEQC, desarrollado por United States Geological Survey.

La Tabla 2 resume los resultados de las simulaciones, con los que se puede analizar cual es la proporción de mezcla más adecuada con el fin de garantizar las concentraciones recomendadas de nutrientes básicos para los cultivos como, $\mathrm{Ca}^{2+}, \mathrm{Mg}^{2+}$ y $\mathrm{SO}_{4}{ }^{2-}$ lo que descartaría la fertilización adicional con dichos elementos. Además se analiza el efecto de las mezclas en las concentraciones de elementos fitotóxicos como $\mathrm{Cl}^{-}, \mathrm{Na}^{+}$y boro, así como su posible afección a cultivos sensibles.

Los resultados ponen de manifiesto que el factor más limitante de la proporción de agua desalinizada en la mezcla es el contenido en boro. Atendiendo a este criterio, la 
máxima proporción para cumplir con las recomendaciones de Yermiyahu et al. (2007) sería de un $40 \%$ para AMD. Si se garantizan unos niveles de boro por debajo de $0,4 \mathrm{mg} \mathrm{L}^{-1}$ a la salida de la planta desalinizadora de Torrevieja (los análisis manejados recogen valores de $0,56 \mathrm{mg} \mathrm{L}^{-1}$ ), las proporciones de mezcla que garantizarían el resto de requerimientos agronómicos se elevan hasta el $50 \%$ de AMD. Además, el agua resultante de la mezcla al $50 \%$ presenta una buena calidad para el riego agrícola, ya que es de menor salinidad que el agua actualmente disponible en La Pedrera y su composición satisface los requerimientos agronómicos planteados en este estudio.Esta estrategia puede permitir un doble objetivo: por una parte disminuir en la medida de las posibilidades los postratamientos de las AMDs para ajustar su calidad a las necesidades de los cultivo y, por otra parte, obtener un menor coste final al de la aplicación directa de AMD.

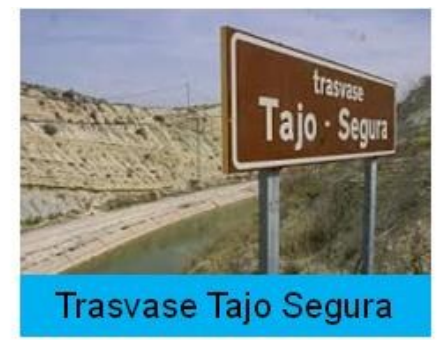

Agua Superficial CSW

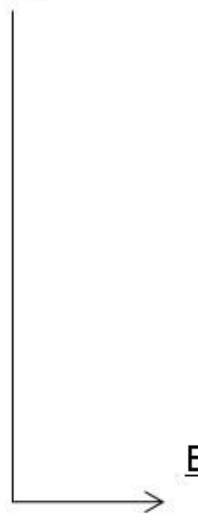

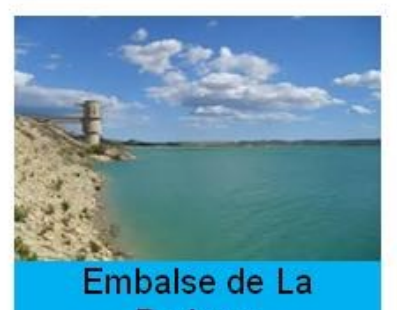

Es:

Escenario 1: CSW + ROSW

Escenario 2: CSW + DSW

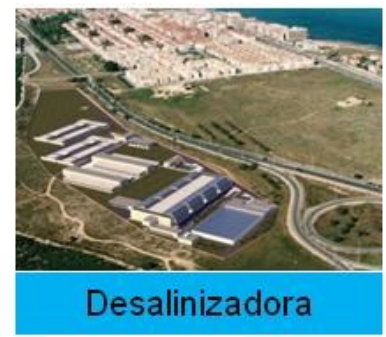

$\underline{\mathrm{Ag}}$

nizadora

upcivires:

1. ROSW

2. DSW

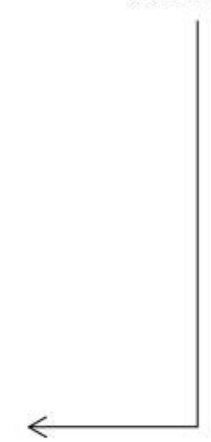

Leyenda:

CSW (Continenta/ Surface Water): Agua de origen superficial.

DSW (Desalinated Sea Water): Agua marina desalinizada por osmosis inversa y remineralizada.

Figura 1. Esquema del sistema de distribución de agua de riego a los usuarios del Canal del Campo de Cartagena y escenarios de mezcla analizados. 
Tabla 2. Composición del agua resultante de la mezcla da AMD en distintas proporciones con el agua que abastece el embalse de La Pedrera.

\begin{tabular}{|c|c|c|c|c|c|c|c|c|c|}
\hline \multirow[t]{2}{*}{ Parámetro } & \multirow[t]{2}{*}{ Unidades } & \multicolumn{8}{|c|}{ Porcentaje de DSW en la mezcla } \\
\hline & & $0 \%$ & $20 \%$ & $40 \%$ & $60 \%$ & $70 \%$ & $80 \%$ & $90 \%$ & $100 \%$ \\
\hline $\mathrm{pH}$ & & 8,4 & 8,4 & 8,4 & 8,4 & 8,4 & 8,4 & 8,4 & 8,3 \\
\hline $\mathrm{CE}_{20}$ & $\mu S \mathrm{~cm}^{-1}$ & 934 & 868 & 799 & 726 & 688 & 648 & 606 & 562 \\
\hline $\mathrm{T}^{\mathrm{a}}$ & ${ }^{\circ} \mathrm{C}$ & 22 & 22 & 21 & 21 & 21 & 20 & 20 & 20 \\
\hline $\mathrm{HCO}_{3}^{-}$ & $\mathrm{mg} \mathrm{L}^{-1}$ & 195 & 162 & 139 & 116 & 105 & 93 & 82 & 71 \\
\hline $\mathrm{Ca}^{2+}$ & $\mathrm{mg} \mathrm{L}^{-1}$ & 98 & 85 & 71 & 57 & 50 & 43 & 36 & 29 \\
\hline $\mathrm{Mg}^{2+}$ & $\mathrm{mg} \mathrm{L}^{-1}$ & 42 & 35 & 27 & 19 & 16 & 12 & 8 & 4 \\
\hline $\mathrm{Na}^{+}$ & $\mathrm{mg} \mathrm{L}^{-1}$ & 49 & 57 & 64 & 72 & 75 & 79 & 82 & 86 \\
\hline $\mathrm{K}^{+}$ & $\mathrm{mg} \mathrm{L}^{-1}$ & 3,3 & 3,4 & 3,5 & 3,7 & 3,7 & 3,8 & 3,8 & 3,9 \\
\hline $\mathrm{Cl}^{-}$ & $\mathrm{mg} \mathrm{L}^{-1}$ & 71 & 86 & 101 & 117 & 124 & 132 & 139 & 147 \\
\hline $\mathrm{SO}_{4}{ }^{2-}$ & $\mathrm{mg} \mathrm{L}^{-1}$ & 279 & 229 & 173 & 119 & 90 & 62 & 34 & 7 \\
\hline $\mathrm{B}^{3+}$ & $\mathrm{mg} \mathrm{L}^{-1}$ & 0,13 & 0,22 & 0,30 & 0,39 & 0,43 & 0,47 & 0,52 & 0,56 \\
\hline STD & $\mathrm{mg} \mathrm{L}^{-1}$ & 647 & 581 & 514 & 447 & 413 & 380 & 346 & 313 \\
\hline $\begin{array}{l}\text { Dureza } \\
\mathrm{CaCO}_{3}\end{array}$ & $\mathrm{mg} \mathrm{L}^{-1}$ & 421 & 355 & 289 & 223 & 190 & 157 & 123 & 90 \\
\hline $\begin{array}{c}\text { Alcalinidad } \\
\mathrm{CaCO}_{3}\end{array}$ & $\mathrm{mg} \mathrm{L}^{-1}$ & 168 & 147 & 125 & 103 & 93 & 82 & 71 & 60 \\
\hline SAR & $\left(\mathrm{mmol} \mathrm{L}^{-1}\right)^{0,5}$ & 1,5 & 1,6 & 2,3 & 2,9 & 3,4 & 3,9 & 4,6 & 5,6 \\
\hline
\end{tabular}

\section{4- Conclusiones y recomendaciones}

Todos los problemas agronómicos y de gestión relacionados con la aplicación de uso $A M D$ al riego agrícola se pueden resolver mediante una correcta regulación de este tipo de suministros, que normalice la calidad a conseguir con los postratamientos de remineralización, y que permita la optimización de su gestión conjunta con la de otros recursos hídricos disponibles en cada zona regable. Para este complejo objetivo se propone la constitución de un comité multidisciplinar que aborde esta cuestión para la singularidad del regadío español, de forma similar a como ya se ha hecho en Israel. El cumplimiento de dicha regulación minimizaría los riesgos de carencias nutricionales y fitotoxicidad; los posibles efectos perjudiciales sobre instalaciones de distribución y de riego; y las necesidades de adaptación de los agricultores y comunidades de regantes.

La necesaria adecuación del AMD a los requerimientos del riego agrícola se puede plantear de tres formas: con postratamientos de remineralización en las plantas desalinizadoras, con la reprogramación de la fertirrigación en parcela, y mediante mezcla con aguas continentales. Varios autores concluyen que si el agua desalinizada se destina a uso agrícola, la mezcla con otras aguas continentales es la estrategia más económica e interesante en la mayoría de los casos. Cuando la mezcla con aguas continentales no es posible, la incorporación de nutrientes en la planta desalinizadora es económica y ambientalmente más ventajosa, implicando además que el desempeño y coste de estos procesos los asume el productor/suministrador del agua desalinizada en lugar del agricultor.

En base a los resultados del caso de estudio, se recomienda que si se pretende consolidar un aporte regular de agua marina desalinizada con las condiciones de calidad 
actuales en la planta de Torrevieja, no se supere el porcentaje del $40 \%$ en la mezcla, porcentaje que se podría elevar al $50 \%$ si se mantiene la concentración de boro en la planta de Torrevieja por debajo de $0,4 \mathrm{mg} \mathrm{L}^{-1}$.

\section{5- Agradecimientos}

Este trabajo sintetiza en contenido del estudio "Antecedentes y problemática de la aplicación de aguamarina desalinizada al riego agrícola", financiado por el Sindicato Central de Regantes del Acueducto Tajo-Segura (SCRATS) y disponible en http://www.scrats.es/memorias-e-informes.html.

\section{6- Bibliografía}

Ayers, R.S., Westcot, D.W., 1985. Water quality for agriculture. FAO Irrigation and Drainage Paper 29. Food and Agriculture Organization of the United Nations, Roma.

Ben-Gal, A., Yermiyahu, U., Cohen, S., 2009. Fertilization and blending alternatives for irrigation with desalinated water. J. Environ. Qual. 38, 529-536.

Birnhack, L., Shlesinger, N., Lahav, O., 2010. A cost effective method for improving the quality of inland desalinated brackish water destined for agricultural irrigation. Desalination 262,152-160.

Díaz, F.J., Tejedor, M., Jiménez, C., Grattanb, S.R., Dorta, M., Hernández, J.M., 2013. The imprint of desalinated seawater on recycled wastewater: Consequences for irrigation in Lanzarote Island, Spain. Agric. Water Manage. 116, 62-72.

Duranceau, S.J., Pfeiffer-Wilder, R.J., Douglas, S.A., Peña-Holt, N., Watson, I.C., 2011. Post-treatment stabilization of desalinated water. Water Research Foundation, Denver.

FAO, 2006. Water desalination for agricultural applications. Proceedings of the FAO Expert Consultation on Water Desalination for Agricultural Applications. Eds. Beltrán JM, KooOshima S. Food and Agriculture Organization, Roma.

Hilal, N., Kim, G.J., Somerfield, C., 2011. Boron removal from saline water: a comprehensive review. Desalination 273, 23-35.

Lahav, O., Birnhack, L., 2007. Quality criteria for desalinated water following post treatment. Desalination 207, 286-303.

Lahav, O., Kochva, M., Tarchitzky, J., 2010. Potential drawbacks associated with agricultural irrigation with treated wastewaters from desalinated water origin and possible remedies. Water Sci. Technol. 61, 2451-2460.

Muyen, Z., Moore, G.A., Wrigley, R.J., 2011. Soil salinity and sodicity effects of wastewater irrigation in South East Australia. Ag. Water Manage. 99, 33-41.

Shaffer, D.L., Yip, N.Y., Gilron, J., Elimelech, M., 2012. Seawater desalination for agriculture by integrated forward and reverse osmosis: Improved product water quality for potentially less energy. J. Membrane Sci. 415-416, 1-8.

Yermiyahu, U., Tal, A., Ben-Gal, A., Bar-Tal, A., Tarchitzky, J., Lahav, O., 2007. Rethinking desalinated water quality and agriculture. Science 318, 920-921.

Zarzo, D., Campos, E., Terrero, P., 2013. Spanish experience in desalination for agriculture. Desalin. Water Treat. 51, 53-66. 